\section{BRCA1 and BRCA2: a common pathway of genome protection but different breast cancer subtypes}

\author{
Simon A. Joosse
}

I read with great interest the recent Opinion article (BRCA1 and BRCA2: different roles in a common pathway of genome protection. Nature Rev. Cancer 12, 68-78 (2012)) ${ }^{1}$ by Roy and colleagues. Both BRCA1 and BRCA2 have vital roles in the same DNA repair pathway, homologous recombination (HR); nevertheless, the pathology and molecular biology of breast tumours that develop in BRCA1-mutation carriers are very different from those of BRCA2 mutation-associated breast tumours. Roy and colleagues provide several interesting theories to explain why $B R C A 1$-mutation carriers predominantly develop oestrogen receptor (ER)-negative tumours, whereas BRCA2-mutation carriers show the same range of molecular subtypes as sporadic breast cancer. In this correspondence, I discuss a plausible explanation for this phenomenon, the cell of origin, based on recent findings.

Mammary stem cells (MaSCs), from which all the mammary epithelial cells originate, are ER-negative and give rise to progenitor cells ${ }^{2}$. Besides its roles in HR and many other processes, BRCA1 is required for the differentiation of progenitor cells to mature ER-positive luminal cells, whereas BRCA2 is not ${ }^{3}$. Consequently, luminal progenitor cells may accumulate in BRCA1-mutation carriers when the wild-type allele is lost, as they are unable to differentiate ${ }^{4}$. Having a defective HR pathway and the ability to selfrenew, these cells are candidates to develop into undifferentiated ER-negative breast can$\mathrm{cer}^{5}$. Thus, because a lack of BRCA1 prevents cell differentiation, it can be assumed that most of the breast tumours that develop in BRCA1-mutation carriers originate from the same cell lineage, making BRCA1-associated cancer a homogeneous tumour group. Conversely, BRCA2-associated breast cancer is a heterogeneous group, showing the same diversity in molecular subtypes as sporadic breast cancer, with similar pathological and molecular characteristics ${ }^{6,7}$; this suggests that its origin is derived from different cell lineages. The average age at which BRCA2-mutation carriers are diagnosed with breast cancer is later than BRCA1 carriers; additionally, the risk of BRCA2-mutation carriers developing breast cancer is significantly lower. This supports the idea that BRCA1-deficient cells have an early advantage in becoming a tumour cell compared with BRCA2-deficient cells; that is, they have the ability to self-renew.

Remarkable similarities between sporadic and hereditary BRCA1-deficient breast cancer indicate that BRCA1 also has an early role in sporadic tumorigenesis ${ }^{8}$. However, it is likely that the loss of BRCA1 expression can occur at any stage of tumour development and in any molecular breast cancer subtype. To test the second explanation of Roy and colleagues - whether BRCA1 influences the transcriptional activity of ER - I reanalysed 51 ER-positive luminal breast tumours from an earlier study ${ }^{8}$. A small group (seven of 51) showed a typical BRCA1-like array-comparative genomic hybridization $(\mathrm{aCGH})$ profile (as described elsewhere ${ }^{9}$ ), of which most (six of seven) showed clear BRCA1 mRNA downregulation, as determined by quantitative PCR. Although further research is required, these results suggest that ER expression is not affected by the absence of BRCA1.

Because of the wide range of differences found between BRCA2- and BRCA1-associated breast tumours, factors other than HR deficiency alone must have important roles in the development of hereditary breast cancer. Of these, the cell of origin might be the most influential factor on the characteristics of a tumour.

Simon A. Joosse is at the Department of Tumor Biology, University Medical Center Hamburg-Eppendorf, Martinistrasse 52, 20246 Hamburg, Germany. e-mail: s.joosse@in-silico.net

doi: 10.1038/nrc3181-c2

Roy, R., Chun, J. \& Powell, S. N. BRCA1 and BRCA2 different roles in a common pathway of genome protection. Nature Rev. Cancer 12, 68-78 (2012).

2. Shackleton, M. et al. Generation of a functional mammary gland from a single stem cell. Nature 439 , 84-88 (2006)

3. Liu, S. et al. BRCA1 regulates human mammary stem progenitor cell fate. Proc. Natl Acad. Sci. USA 105, 1680-1685 (2008).

4. Lim, E. et al. Aberrant luminal progenitors as the candidate target population for basal tumor development in BRCA1 mutation carriers. Nature Med. 15, 907-913 (2009).

5. Molyneux, G. et al. BRCA1 basal-like breast cancers originate from luminal epithelial progenitors and not from basal stem cells. Cell Stem Cell 7, 403-417 (2010).

6. Jonsson, G. et al. Genomic subtypes of breast cancer identified by array-comparative genomic hybridization display distinct molecular and clinical characteristics. Breast Cancer Res. 12, R42 (2010).

7. Joosse, S. A. et al. Prediction of BRCA2-association in hereditary breast carcinomas using array-CGH. Breast Cancer Res. Treat. 132, 379-389 (2012).

8. Joosse, S. A. et al. Genomic signature of BRCA1 deficiency in sporadic basal-like breast tumors. Genes Chromosom. Cancer. 50, 71-81 (2011).

9. Joosse, S. A. et al. Prediction of BRCA1-association in hereditary non-BRCA $1 / 2$ breast carcinomas with arrayCGH. Breast Cancer Res. Treat. 116, 479-489 (2009).

Competing interests statement

S.A.J. is a named contributor on the patent for the array-CGH classifier referred to in this correspondence. 